\title{
Avaliação da força de preensão palmar e dos volumes pulmonares de pacientes hospitalizados por condições não cirúrgicas
}

\author{
Evaluation of handgrip strength and lung volumes inpatients hospitalized for \\ nonsurgical conditions
}

\author{
Dulciane Nunes Paiva1, Diogo Fanfa Bordin², Ricardo Gass 3 , Regis Jean Severo ${ }^{4}$, Natália Rodrigues Brum4, \\ Camila da Cunha Niedermeyer ${ }^{4}$, Michele Saldanha ${ }^{4}$, Angélica Figueiró Olivero ${ }^{4}$, Larissy dos Santos Americo ${ }^{4}$, \\ Emeline Rässier Schafer ${ }^{4}$, Mônica Wietzke ${ }^{5}$, Silvia Isabel Rech Franke ${ }^{6}$, Dannuey Machado Cardoso
}

\begin{abstract}
${ }^{1}$ Doutora em Ciências Médicas pela Universidade Federal do Rio Grande do Sul (UFRGS), Docente do Curso de Fisioterapia e do Programa de Pós-Graduação em Promoção da Saúde da Universidade de Santa Cruz do Sul (UNISC), Santa Cruz do Sul, RS; ${ }^{2}$ Bolsista de Iniciação Científica PROBIC/FAPERGS, Acadêmico do Curso de Fisioterapia da UNISC, Santa Cruz do Sul, RS; ${ }^{3}$ Bolsista de Iniciação Científica PIBIC/CNPq, Acadêmico do Curso de Fisioterapia da UNISC, Santa Cruz do Sul, RS; ${ }^{4}$ Bolsistas do Programa Voluntário de Bolsas de Iniciação Científica (PUIC), Acadêmicos do Curso de Fisioterapia da UNISC, Santa Cruz do Sul, RS; ${ }^{5}$ Profissional de Educação Física, Mestre do Programa de Pós-Graduação em Promoção da Saúde da UNISC, Santa Cruz do Sul, RS; ${ }^{6}$ Doutora em Biologia Celular e Molecular pela UFRGS, Docente do Curso de Nutrição e do Programa de Pós-Graduação em Promoção da Saúde da UNISC, Santa Cruz do Sul, RS; ${ }^{7}$ Mestre em Ciências Médicas pela UFRGS, Docente do Curso de Fisioterapia da UNISC, Santa Cruz do Sul, RS.
\end{abstract}

Estudo conduzido com recursos da Fundação de Amparo à Pesquisa do Estado do Rio Grande do Sul (FAPERGS) e do Conselho Nacional de Desenvolvimento Científico e Tecnológico (CNPq).

\section{RESUMO}

Objetivos: Avaliar a força de preensão palmar e os volumes pulmonares de indivíduos hospitalizados.

Métodos: Este estudo transversal avaliou indivíduos com idades entre 30 a 80 anos, de ambos os gêneros e hospitalizados sob condição não cirúrgica por pelo menos 48 horas (Grupo Hospitalizado) e indivíduos hígidos, acessados em um clube esportivo (Grupo Controle), em Santa Cruz do Sul, Estado do Rio Grande do Sul. Foi realizada espirometria para obtenção da capacidade vital forçada, volume expirado forçado no primeiro segundo, relação volume expiratório forçado no primeiro segundo/capacidade vital forçada, pico de fluxo expirado e do fluxo expiratório forçado entre $25 \%$ e $75 \%$ da capacidade vital forçada. Para avaliação da força de preensão palmar foi utilizada Dinamometria Manual.

Resultados: Foram avaliados 32 indivíduos, sendo $16 \mathrm{em}$ cada grupo. Houve homogeneidade quanto à idade $(\mathrm{p}=0,183)$, altura $(\mathrm{p}=0,685)$, peso $(\mathrm{p}=0,105)$ e índice de massa corpórea $(\mathrm{p}=0,157)$ entre os dois grupos. O Grupo Hospitalizado apresentou mediana de internação de 9,5 dias (7-15; mínimo 3, máximo 17). Em comparação ao Grupo Controle, o Grupo Hospitalizado apresentou redução na capacidade vital forçada $(p<0,001)$, volume expirado forçado no primeiro segundo $(p<0,001)$, relação volume expiratório forçado no primeiro segundo/capacidade vital forçada $(p=0,006)$, pico de fluxo expirado $(p<0,001)$ e fluxo expiratório forçado entre $25 \%$ e $75 \%$ da capacidade vital forçada ( $<<0,001)$, bem como na força de preensão palmar $(\mathrm{p}=0,001)$.

Conclusões: Os indivíduos hospitalizados apresentaram redução da força de preensão palmar e dos volumes pulmonares.

DESCRITORES: HOSPITALIZAÇÃO; FORÇA MUSCULAR; FORÇA DA MÃO; ESPIROMETRIA.

\section{ABSTRACT}

Aims: The aim of this investigation was to analyzehandgrip strength and lung volumes in hospitalized patients.

Methods: This cross-sectional study enrolled subjects aged between 30 and 80 years of both genders and hospitalized under non-surgical conditions for at least 48 hours (Hospitalized Group) and healthy subjects accessed in a sport club (Control Group) in Santa Cruz do Sul, Rio Grande do Sul state, Brazil. Forced vital capacity, expiratory volume in the first second, expiratory volume in the first second/forced vital capacity relation, expiratory flow peak, and forced expiratory flow between $25 \%$ and $75 \%$ of vital capacity were evaluated. The handgrip strength was evaluated through hand dynamometry.

Results: A total of 32 subjects were evaluated, being 16 in each group. They matched in age $(\mathrm{p}=0.183)$, height $(\mathrm{p}=0.685)$, weight $(\mathrm{p}=0.105)$ and body index mass $(\mathrm{p}=0.157)$ in both groups. The Hospitalized Group had a median hospital stay of 9.5 days $(7-15$; minimum 3, maximum 17). When compared to the Control Group, the Hospitalized Group had a reduction in forced vital capacity ( $<<0.001)$, expiratory volume in the first second $(\mathrm{p}<0.001)$, expiratory volume in the first second / forced vital capacity relation $(\mathrm{p}=0.006)$, peak expiratory flow ( $<0.001)$ and forced expiratory flow between $25 \%$ and $75 \%$ of vital capacity $(p<0.001)$, as well as in the handgrip force $(p=0.001)$.

Conclusions: The hospitalized subjects showed decrease in handgrip and lung volumes.

KEY WORDS: HOSPITALIZATION; MUSCLE STRENGTH; HAND STRENGHT; SPIROMETRY. 


\section{INTRODUÇÃO}

A hospitalização é considerada de grande risco para jovens e para idosos, ${ }^{1,2}$ devendo-se considerar que os efeitos desta envolvem a hipoventilação, principalmente devido à condição prévia de saúde do indivíduo, e também à disfunção diafragmática temporária, causada por longos períodos em decúbito dorsal, ${ }^{3}$ depleção nutricional ${ }^{4}$ e perda de capacidade funcional. ${ }^{5}$

Sabe-se que adultos apresentam maior número de internações hospitalares, porém pessoas com 80 anos ou mais de idade gastam cinco vezes mais com a hospitalização. ${ }^{6-9}$ A restrição ao leito induz a alterações no humor, na força, na coordenação motora e na tolerância aos esforços, impondo restrições às atividades de autocuidado, sendo tais achados aplicáveis principalmente aos idosos. ${ }^{10}$ Além desses efeitos, a hospitalização pode ocasionar também redução da capacidade funcional, resultando em perda da força muscular, inclusive dos músculos respiratórios, e isso se deve ao tempo de imobilização e repouso prolongado no leito. Este fato afeta todos os sistemas do organismo, podendo causar complicações definidas como Síndrome da Imobilização, que se agravam quando associadas à condição prévia de saúde dos indivíduos, provocando deterioração funcional e perda de independência para a execução de atividades da vida diária, ${ }^{2,11,12}$ culminando em aumento no tempo de internação, mudança na qualidade de vida e institucionalização. ${ }^{6,13}$

Com a hospitalização, ocorrem ainda modificações no sistema respiratório que englobam a redução da complacência da parede torácica e dos volumes pulmonares. ${ }^{14,15}$ Embora as doenças respiratórias sejam muito prevalentes, há escassez de relatos na literatura sobre o efeito da internação hospitalar sobre os volumes pulmonares.

Os testes de função pulmonar são indicados para diagnosticar as disfunções ventilatórias bem como para classificar o estadiamento de doenças pulmonares. ${ }^{16}$, ${ }^{17}$ A espirometria possibilita a avaliação dos volumes pulmonares dinâmicos. As medidas da capacidade vital e dos demais volumes pulmonares são úteis no monitoramento da função pulmonar, indicando a gravidade das disfunções pulmonares. ${ }^{18} \mathrm{~A}$ avaliação da força muscular periférica através da dinamometria manual surge como uma alternativa simples, objetiva, de baixo custo e não invasiva, para aferição da força voluntária máxima depreensão palmar, e tem como princípio estimar a força muscular. Tal teste possibilita identificar riscos à saúde relacionados à força muscular, sendo considerado um bom indicador da força muscular global e também permite avaliar o desempenho físico em diversas situações clínicas. ${ }^{19}$

Dessa forma, as estratégias para conservação da funcionalidade e prevenção de eventos adversos durante a internação precisam ser seguidas por uma equipe multidisciplinar para impedir que a hospitalização se torne motivo de incapacidade. ${ }^{6,20}$ É importante também observar o prejuízo causado pela hospitalização sobre a capacidade funcional e a função pulmonar, para que se desenvolvam estratégias para minimizar os efeitos deletérios desta.

O objetivo do presente estudo foi avaliar a força de preensão palmar (FPP) e os volumes pulmonares de indivíduos hospitalizados e estabelecer possível correlação entre o tempo de internação hospitalar e os volumes pulmonares.

\section{MÉTODOS}

Foi conduzido um estudo transversal na cidade de Santa Cruz do Sul, estado do Rio Grande do Sul, onde a amostra foi constituída por pacientes admitidos nas enfermarias do Hospital de Santa Cruz (Grupo Hospitalizado-GH) e indivíduos hígidos, sedentários, voluntários ao estudo (Grupo Controle - GC), tendo o estudo sido aprovado pelo Comitê de Ética em Pesquisa da Universidade de Santa Cruz do Sul.

\section{Critérios de inclusão e exclusão}

Para admissão ao GH foram incluídos indivíduos com idade entre 30 e 80 anos, admitidos ao hospital devido a eventos clínicos agudos ou crônicos agudizados, secundários a intervenções não cirúrgicas, com tempo de internação maior que 48 horas, acamados, porém que pudessem responder aos questionamentos, bem como compreender e aceitar as informações contidas no Termo de Consentimento Pós-Informado. Foram excluídos aqueles que apresentassem índice de massa corporal (IMC) $>40 \mathrm{klg} / \mathrm{m}^{2}$, histórico de instabilidade hemodinâmica, ${ }^{21}$ pneumopatias agudas ou crônicas e qualquer procedimento cirúrgico na hospitalização. Os indivíduos do $\mathrm{GH}$ não haviam recebido nenhuma intervenção fisioterapêutica até o momento da avaliação.

Para participarem do GC foram incluídos indivíduos hígidos de ambos os gêneros, recrutados no Clube Poliesportivo de Santa Cruz do Sul, sem história prévia de hospitalização nos últimos cinco anos. Foram excluídos indivíduos com IMC $>40 \mathrm{~kg} / \mathrm{m}^{2}$; praticantes de atividades físicas regulares três vezes ou mais por 
semana; e os que tivessem apresentado problemas respiratórios no período de 30 dias previamente às avaliações.

\section{Variáveis}

Foram avaliados gênero, idade, IMC, saturação periférica de oxigênio $\left(\mathrm{SpO}_{2}\right)$, frequência cardíaca (FC), frequência respiratória (FR), pressão arterial (PA), tempo total de internação, FPP no membro dominante e volumes pulmonares. Para calcular o IMC foi necessário utilizar o método indireto ${ }^{22}$ devido ao fato dos pacientes encontrarem-se acamados, que tornava inviável sua retirada do leito para a mensuração do peso e altura.

\section{Avaliação dos volumes pulmonares}

Os volumes pulmonares foram avaliados através de espirometria digital (EasyOne ${ }^{\circledR}$, Modelo 2001, Zurique, Suíça) onde foram obtidas as variáveis: capacidade vital forçada (CVF), volume expiratório forçado no primeiro segundo $\left(\mathrm{VEF}_{1}\right)$, relação $\mathrm{VEF}_{1} / \mathrm{CVF}$, pico de fluxo expiratório (PEF), fluxo expiratório forçado entre $25 \%$ e $75 \%$ da CVF $\left(\mathrm{FEF}_{25-75 \%}\right)$ em três manobras, ${ }^{22}$ sendo a melhor validada automaticamente pelo dispositivo e os valores expressos através de valores preditos para a população brasileira. ${ }^{23,24}$

\section{Avaliação da força de preensão palmar}

A avaliação da FPP foi realizada conforme as recomendações da The American Society of Hands Theraphists (ASHT) através de Dinamômetro Manual (Jamar ${ }^{\circledR}$, modelo Adjustable Dynamometer, Califórnia, Estados Unidos da América) com os pacientes posicionados sentados, com ombro aduzido e neutramente rodado, cotovelo flexionado a $90^{\circ}$, antebraço em posição neutra de prono-supinação e articulação do punho entre $0^{\circ}$ e $30^{\circ}$ de extensão. ${ }^{25} \mathrm{As}$ manobras foram repetidas três vezes, sendo calculada a média entre as medidas e dado um tempo de descanso de 60 segundos entre uma medida e outra. Os valores obtidos foram comparados como previsto na metodologia. ${ }^{26}$

\section{Análise estatística}

O programa Statistical Package for the Social Sciences (SPSS, versão 20.0) foi utilizado para tratamento dos resultados, que foram apresentados em média e desvio padrão (DP) ou mediana e intervalo interquartil, sendo a normalidade averiguada pelo teste de Shapiro-Wilk. O teste qui-quadrado foi utilizado para comparar as proporções de gênero e indivíduos idosos entre os grupos. A associação do tempo de internação com os volumes pulmonares foi avaliada através do teste de Correlação de Spearman. Para a análise dos volumes pulmonares e FPP utilizou-se o teste $t$ de Student para amostras independentes. Foi considerado significativo um $\mathrm{p}<0,05$.

\section{RESULTADOS}

A amostra foi composta por 32 participantes $(\mathrm{GH}$, $\mathrm{n}=16$; GC n=16), havendo distribuição homogênea tanto para gênero quanto para as características antropométricas e sinais vitais entre os grupos (Tabela 1). A mediana do tempo total de internação hospitalar dos pacientes do GH foi de 9,5 dias (7-15; mínimo 3 , máximo 17) tendo as medidas sido realizadas em uma mediana de tempo de internação de 5,4 dias (3,2-7,0; mínimo 2, máximo 10). As causas de internação foram pancreatite aguda, trauma em região lombar, leptospirose, infarto agudo do miocárdio, úlcera varicosa infectada, erisipela, pé diabético infectado, ansiedade generalizada, doença de Crohn, candidíase esofágica, cetoacidose diabética e neurotoxoplasmose. Houve diferença entre os grupos quanto à $\mathrm{SpO}_{2}$, que se apresentou reduzida no $\mathrm{GH}$, porém não o suficiente para caracterizar dessaturação.

Tabela 1. Características na linha de base dos indivíduos hospitalizados $(\mathrm{GH})$ quando comparados aos não hospitalizados (GC).

\begin{tabular}{|c|c|c|c|}
\hline Variáveis & GC $(n=16)$ & $\mathrm{GH}(n=16)$ & p \\
\hline \multicolumn{4}{|l|}{ Gênero } \\
\hline Masculino, n (\%) & $3(18,8)$ & $8(50)$ & 0,063 \\
\hline \multicolumn{4}{|l|}{ Características Antropométricas } \\
\hline Idade (anos) & $59,8 \pm 9,2$ & $53,8 \pm 14,9$ & 0,183 \\
\hline PesO $_{\text {estimado }}(\mathrm{kg})$ & $70 \pm 17,7$ & $61 \pm 12,3$ & 0,105 \\
\hline Altura $_{\text {estimada }}(\mathrm{cm})$ & $162,2 \pm 8,2$ & $161,1 \pm 7,5$ & 0,685 \\
\hline $\mid \mathrm{MC}_{\text {estimado }}\left(\mathrm{kg} / \mathrm{m}^{2}\right)$ & $26 \pm 5,7$ & $23,4 \pm 4$ & 0,157 \\
\hline \multicolumn{4}{|l|}{ Sinais Vitais } \\
\hline $\begin{array}{l}\text { Pressão arterial sistólica } \\
\text { (mm Hg) }\end{array}$ & $121,8 \pm 12,7$ & $126,25 \pm 13,1$ & 0,346 \\
\hline $\begin{array}{l}\text { Pressão arterial diastólica } \\
(\mathrm{mm} \mathrm{Hg})\end{array}$ & $73,1 \pm 19,5$ & $81,2 \pm 10,2$ & 0,152 \\
\hline Frequência cardíaca (bpm) & $75,8 \pm 5,7$ & $77,3 \pm 11,9$ & 0,642 \\
\hline Frequência respiratória (irpm) & $16,8 \pm 3,6$ & $19,1 \pm 3,5$ & 0,087 \\
\hline $\mathrm{SpO}_{2}(\%)$ & $97,1 \pm 1,3$ & $94,5 \pm 3,5$ & $0,010^{*}$ \\
\hline
\end{tabular}

$\mathrm{GC}=$ grupo controle (indivíduos hígidos não hospitalizados); $\mathrm{CH}=$ grupo hospitalizado (indivíduos hospitalizados por condições não cirúrgicas); IMC = índice de massa corporal; $\mathrm{SpO}_{2}=$ saturação periférica de oxigênio.

Valores expressos em média \pm desvio padrão, exceto para o gênero.

* Significância estatística $(p<0,05)$. 
Os volumes pulmonares em relação ao predito mostram-se significativamente reduzidos no $\mathrm{GH}$ em relação ao $\mathrm{GC}$, tanto para CVF como para $\mathrm{VEF}_{1}$, relação $\mathrm{VEF}_{1} / \mathrm{CVF}$, PFE e $\mathrm{FEF}_{25-75 \%}$. Ressalta-se que houve diferença estatística nos volumes pulmonares entre os dois grupos (Figura 1).

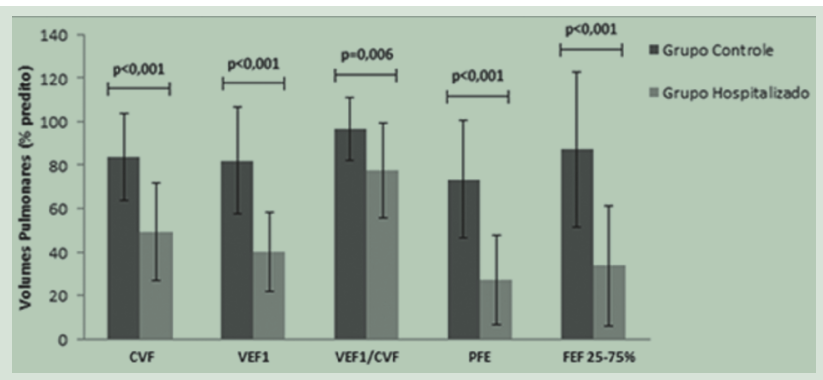

$\mathrm{CVF}=$ capacidade vital forçada; $\mathrm{VEF}$ volume expiratório forçado no primeiro segundo; $\mathrm{PEF}=$ pico de fluxo expiratório; FEF $25-75 \%$ = fluxo expiratório forçado entre $25 \%$ e $75 \%$ da CVF.

Figura 1. Comparação dos volumes pulmonares entre os grupos (controle e hospitalizado). Teste t de Student para amostras independentes $(\mathrm{p}<0,05)$.

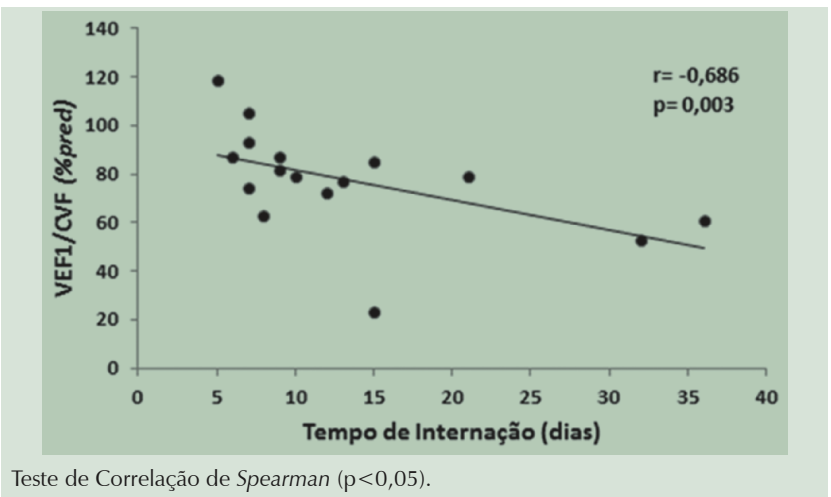

Figura 2. Correlação entre o tempo total de internação dos pacientes hospitalizados e a relação volume expiratório forçado no primeiro segundo/capacidade vital forçada $\left(\mathrm{VEF}_{1} / \mathrm{CVF}\right)$.

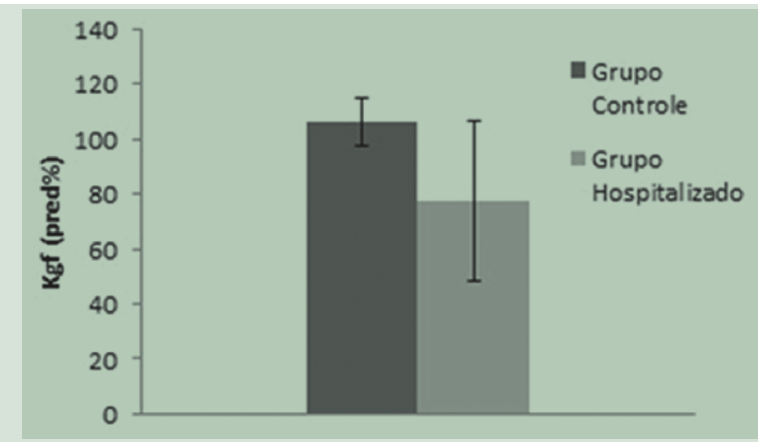

Teste t de Student para amostras independentes $(\mathrm{p}<0,05)$.

Figura 3. Força de preensão palmar (kgf) dos indivíduos hospitalizados (Grupo Hospitalizado) quando comparados aos não hospitalizados (Grupo Controle).
Ao correlacionar o tempo total de internação com os volumes pulmonares, observamos que o mesmo apresentou correlação significativa apenas com a relação $\mathrm{VEF}_{1} / \mathrm{CVF}$, sendo esta de caráter inverso (Figura 2). Foi avaliada a FPP de membro dominante para analisar a força muscular global, sendo constatada diferença estatística entre os grupos $(\mathrm{p}<0,001)$, com redução desta variável no GH (Figura 3).

\section{DISCUSSÃO}

O presente estudo demonstrou que os indivíduos hospitalizados apresentaram redução de todos os volumes pulmonares, bem como da força muscular periférica de membro superior dominante, quando comparados aos indivíduos hígidos.

A identificação de déficits físicos funcionais se faz importante em pacientes hospitalizados sob situação não cirúrgica, pois a mesma é seguida comumente por redução da capacidade funcional e perda de força muscular devido ao prolongado tempo de repouso e imobilismo ao leito. ${ }^{11} \mathrm{O}$ músculo é o mais mutável tecido dentre todos os tecidos biológicos do organismo, onde no repouso completo e prolongado, pode perder de 10 a $15 \%$ de força por semana, chegando até $50 \%$ em até cinco semanas, sendo que esta resposta morfológica é dependente das demandas empregadas. ${ }^{27}$ Tal afirmação pode justificar o fato dos indivíduos hospitalizados terem apresentado redução significativa na FPP, visto que na condição de decúbito prolongado ao leito, a atividade muscular e a força gravitacional exercida sobre ossos e tecidos de apoio são mínimas. ${ }^{28}$

A força de preensão palmar é um importante indicador de declínio do status nutricional ${ }^{29}$ e também um bom indicador da capacidade funcional ${ }^{30} \mathrm{e}$ tem sido utilizada em diversas situações clínicas como para correlacionar índices de mortalidade em pacientes na lista de espera para o transplante de fígado, ${ }^{31}$ correlacionar a força de preensão palmar e a espasticidade em pacientes hemiparéticos após acidente vascular cerebral ${ }^{32} \mathrm{e}$ para analisar a força e a resistência em escaladores esportivos..$^{33}$

$\mathrm{O}$ posicionamento de repouso do paciente em decúbito dorsal provoca redução dos volumes e capacidades pulmonares e aumento do trabalho respiratório e cardíaco. Sabe-se que a imobilidade no leito gera redução na força muscular respiratória devido ao desfavorecimento na posição diafragmática e acarreta modificações na estrutura torácica que restringem a expansão pulmonar, provocando diminuição da capacidade inspiratória e enfraquecimento dos 
músculos intercostais e do diafragma, sendo tais eventos observados especialmente em idosos. ${ }^{34,35}$

Em nosso estudo, foi evidenciada redução de todos os volumes pulmonares no grupo de indivíduos hospitalizados e é bem descrito na literatura que a redução dos volumes pulmonares contribui para alterações nas trocas gasosas, resultando em hipoxemia e diminuição na capacidade de difusão, ${ }^{36}$ entretanto, em nosso estudo a $\mathrm{SpO}_{2}$ no grupo de indivíduos hospitalizados se encontrou reduzida em relação aos indivíduos normais, sem ser evidenciado hipoxemia. Deve ser ressaltado que o grupo controle apresentou volumes pulmonares também reduzidos, porém todos os testes foram reprodutíveis em tal grupo. Inferimos que esta seja uma característica intrínseca da amostra estudada, provavelmente devido a perdas provocadas pela idade próxima dos 60 anos no grupo controle.

$\mathrm{O}$ quadro de disfunção pulmonar é encontrado em muitos estudos que envolvem situações pós-operatórias cardíacas ou pulmonares, caracterizando-se por volumes pulmonares reduzidos e respiração superficial, podendo ou não evoluir para complicações pulmonares. ${ }^{37}$ Entretanto, há escassez de relatos na literatura sobre o efeito da internação hospitalar sobre os volumes pulmonares em pacientes não cirúrgicos, devendo ser considerado que a patologia de base, as comorbidades e as doenças nosocomiais, além do imobilismo no leito durante a internação, também podem influir na mecânica ventilatória, induzindo mudanças na estrutura torácica que restringem secundariamente a expansão pulmonar, provocando danos à função pulmonar. Tanto imediatamente, quanto, principalmente, durante prolongado tempo em posição supina, há um deslocamento em direção cranial do diafragma, presumivelmente por consequência do peso visceral e redução do tônus de repouso diafragmático, enfraquecendo este músculo e levando a uma redução do volume da caixa torácica. ${ }^{38}$

Estudos sobre o comando neural diafragmático evidenciam aumento da atividade muscular em cerca de quatro a cinco vezes após a mudança de postura supina para ortostática. ${ }^{39}$ Isto sugere que o comando neural aumenta consideravelmente para compensar as mudanças de cargas impostas pela gravidade quando em ortostase e, da mesma forma, reduz quando em posição supina, como por exemplo, em situações de imobilismo prolongado ao leito, onde ainda se tem associado o deslocamento diafragmático em direção cranial devido ao peso visceral, o qual devido aos fatores supracitados leva à redução dos volumes pulmonares, corroborando com os achados em nosso estudo. ${ }^{40}$
O tempo de internação hospitalar em nosso estudo, segundo dados do Sistema de Informações Hospitalares do Sistema Único de Saúde, é considerado moderado a alto, visto que o número de internações hospitalares de indivíduos acima de 30 anos no município de Santa Cruz do Sul, no período de janeiro a outubro de 2012, foi de 6.426, com média de permanência de 4,4 dias por internação. As causas mais frequentes das internações nessa população foram neoplasias (1.308 casos), distúrbios do aparelho circulatório (1.086 casos) e distúrbios do aparelho respiratório (794 casos). ${ }^{41}$ Foi evidenciada correlação negativa entre o tempo de internação hospitalar e a relação $\mathrm{VEF}_{1} / \mathrm{CVF}$, o que corrobora a inferência de que os pacientes que apresentaram menores índices da relação $\mathrm{VEF}_{1} / \mathrm{CVF}$ permaneceram maior tempo hospitalizados.

Entretanto, nosso estudo apresentou como principal limitação o fato de termos utilizado como valores de referência, para avaliação da força de preensão palmar, dados de origem espanhola, pois estudos desenvolvidos no Brasil contemplam apenas idade entre os 25 e 59 anos, não sendo possível, assim, estabelecer valores preditivos para toda a nossa amostra, que variou entre 30 e 80 anos de idade. Outro fator e, talvez, o mais importante a ser discutido, é o fato de não ter sido possível avaliar os participantes do estudo em sua condição pré-hospitalização, visto que não possuímos um sistema de acompanhamento domiciliar abrangente, o que seria ideal para poder comparar exatamente os reais efeitos da hospitalização sobre volumes pulmonares e força muscular periférica de membro superior.

Os achados deste estudo podem ter implicações práticas quanto à necessidade de se evitar o imobilismo no leito, sendo que a assistência fisioterapêutica é de extrema importância para esse fim. A mobilização precoce no paciente hospitalizado é muito estudada dentro das Unidades de Terapia Intensiva e objetiva evitar riscos de hospitalização prolongada e principalmente a prevenção da fraqueza muscular generalizada, bem como reduzir o tempo de ventilação mecânica, ${ }^{42}$ entretanto há escassez de estudos sobre a mobilização precoce em pacientes não críticos, o que também deve ser estudado com objetivo de se reduzir os prejuízos funcionais dos pacientes, bem como o tempo de hospitalização e os gastos gerados aos sistemas de saúde.

A partir dos resultados expostos pode-se concluir que a situação de hospitalização prolongada está associada à redução dos volumes pulmonares e à perda da força de preensão palmar. Chama-se atenção para a correlação negativa entre $\mathrm{oVEF}_{1}$ e o tempo 
de internação dos pacientes (em nossa amostra os pacientes que permaneceram hospitalizados por mais tempo apresentaram menor $\mathrm{VEF}_{1}$ no momento da avaliação), o que leva a considerar que o efeito do tempo de hospitalização pode ser cumulativo.
Enfatizamos que ações multidisciplinares podem ser tomadas no sentido de evitar a deterioração observada neste estudo, no que tange ao quadro funcional dos pacientes, independentemente de sua condição clínica.

\section{REFERÊNCIAS}

1. Volpato S, Cavalieri M, Guerra G, Sioulis F, Ranzini M, Maraldi C, et al. Performance-based functional assessment in older hospitalized patients: feasibility and clinical correlates. J Gerontol A Biol Sci Med Sci. 2008;63(12):1393-8.

2. de Vos AJ, Asmus-Szepesi KJ, Bakker TJ, de Vreede PL, van Wijngaarden JD, Steyerberg EW, et al. Integrated approach to prevent functional decline in hospitalized elderly: the Prevention and Reactivation Care Program (PReCaP). BMC Geriatr. 2012;12(7):1-11.

3. Delgado P, Lunardi A. Complicações Respiratórias pós-operatórias em Cirurgia Bariátrica: Revisão da Literatura. Fisioter pesq. 2011;18(4):388-92.

4. Oliveira L, Rocha A, Silva J. Avaliação Nutricional em pacientes Hospitalizados: Uma responsabilidade Interdisciplinar. Saber Científico. 2008;1(1):240-52.

5. Sandoval L, Varela L. Estudio comparativo de funcionalidade em pacientes adultos mayores ambulatórios y hospitalizados. Rev Med Hered. 1998;9(4):138-42

6. Sales MVC, Silva TJA, Gil Jr LA, Filho WJ. Efeitos adversos da internação hospitalar para o idoso. Geriatria \& Gerontologia. 2011;4(4): $238-46$

7. Buurman BM, van Munster BC, Korevaar JC, de Haan RJ, de Rooij SE. Variability in measuring (instrumental) activities of daily living functioning and functional decline in hospitalized older medical patients: a systematic review. J Clin Epidemiol. 2011;64(6):619-27.

8. Chou CH, Hwang CL, Wu YT. Effect of exercise on physical function, daily living activities, and quality of life in the frail older adults: a meta-analysis. Arch Phys Med Rehabil. 2012;93(2):237-44.

9. Santos KAd, Koszuoski R, Dias-da-Costa JS, Pattussi MP. Factors associated with functional incapacity among the elderly in Guatambu, Santa Catarina State, Brazil. Cad Saúde Pública. 2007;23(11):2781-88

10. Jorge LL, Sugawara AT, Carneiro MSO. O paciente internado em um hospital geral encaminhado à Medicina Física: perfil epidemiológico e nível funcional. Acta Fisiatr. 2006;13(3):124-9.

11. Reis J, Dantas M, Silva C, Valverde L, Landeiro R. Caracterização da Força Muscular Respiratória e da Capacidade Funcional de Pacientes Internados em uma Enfermaria. Rev Fisioter S Fun. 2012;1(2):3-9.

12. Jorge L, Sugawara A, Carneiro M. O paciente internado em um Hospital Geral encaminhado à Medicina Física: Perfil Epidemiológico e Nível Funcional. Acta Fisiatr. 2006;13(3):124-9.

13. Menezes C, Oliveira VRC, Menezes RL. Repercussões da hospitalização na Capacidade Funcional de idosos. Rev Movimenta. 2010;3(2): 76-84.

14. Trindade TM, Gonçalves MP, Vogt MSL, Schwanz CC, Gomes AT, Marques MR, et al. Capacidade Pulmonar em Idosos praticantes de Hidroginástica. Est Interdisc Envelh. 2011;16(1):79-96.

15. Choi HC, Son KY, Cho B, Park SM, Cho SI. An implication of the short physical performance battery (SPPB) as a predictor of abnormal pulmonary function in aging people. Arch Gerontol Geriatr. 2012;54(3):448-52.

16. Global Strategy for the Diagnosis, Management and Prevention of COPD. Global Initiative for Chronic Obstructive Lung Disease (GOLD) 2014. (Updated Nov 2013). [Citado 18 outubro 2013]. Disponível em: http://www.goldcopd.org/guidelines-global-strategy-for-diagnosismanagement.html

17. Rodrigues MT, Fiterman-Molinari D, Barreto SSM, Fiterman J. O papel da razão FEF50\%/0,5CVF no diagnóstico dosdistúrbios ventilatórios obstrutivos. J Pneumol. 2010;36(1):44-50.

18. Fiore EG, Vieira VL, Cervato AM, Tucilo DR, Cordeiro AdA. Nutritional profile of elderly who frequent a health service basic unit. Rev Cienc Med (Campinas). 2006;15(5):369-77.

19. Martin F, Nebuloni C, Najas M. Correlação entre Estado Nutricional e Força de Preensão Palmar em idosos. Rev Bras Geriatr Gerontol. 2012;15(3):593-604.

20. Lang PO, Meyer N, Heitz D, Drame M, Jovenin N, Ankri J, et al. Loss of independence in Katz's ADL ability in connection with an acute hospitalization: early clinical markers in French older people. Eur J Epidemiol. 2007;22(9):621-30.

21. Rezende E, Rea-Neto A, David CM, Mendes CL, Dias FS, Schettino G, et al. Consenso Brasileiro de Monitorização e Suporte Hemodinâmico - Parte I: Método e Definições. I Simposio Internacional de Monitorização em UTI: RBTI. 2005;17(4):278-81.

22. Chumlea WC, Roche AF, Steinbaugh ML. Estimating stature from knee height for persons 60 to 90 years of age. J Am Geriatr Soc. $1985 ; 33(2): 116-20$.

23. Pereira C. Espirometria. J Pneumol. 2002;28(Suppl 3):S1-S82. 
24. Pereira CAdC, Barreto SdP, Simöes JG, Pereira FWL, Gerstler JG, Nakatani J. Valores de referência para a espirometria em uma amostra da populaçäo brasileira adulta. J Pneumol. 1992;18(1):10-22.

25. Soares A, Júnior J, Fachini J, Domenech S, Júnior N. Correlação entre os testes de Dinamometria de Preensão Manual, Escapular e Lombar. Rev Acta Bras Mov Hum. 2012;2(1):65-72.

26. Luna-Heredia E, Martin-Pena G, Ruiz-Galiana J. Handgrip dynamometry in healthy adults. Clin Nutr. 2005;24(2):250-8.

27. Carvalho C, Shimano A, Volpon J. Efeito da Imobilização e do exercício físico em algumas propriedades mecânicas do músculo esquelético. Rev Bras Eng Biomed. 2002;18(2):65-73.

28. Boechat J, Manhães F, Filho R, Istoé R. A síndrome do imobilismo e seus efeitos sobre o aparelho locomotor do idoso. InterScience Place. 2012;1(5):89-107.

29. Humphreys J, de la Maza P, Hirsch S, Barrera G, Gattas V, Bunout D. Muscle strength as a predictor of loss of functional status in hospitalized patients. Nutrition. 2002;18(7-8):616-20.

30. Pieterse S, Manandhar M, Ismail S. The association between nutritional status and handgrip strength in older Rwandan refugees. Eur J Clin Nutr. 2002;56(10):933-9.

31. Carvalho E, Isem M, Lima P, Machado C, Biagini A, Massarollo P. Força muscular e mortalidade na lista de espera de transplante de fígado. Rev Bras Fisioter. 2008;12(3):235-40.

32. Paz L, Marães V, Borges G. Relação entre a força de preensão palmar e a espasticidade em pacientes hemiparéticos após acidente vascular cerebral. Acta Fisiátrica. 2011;18(2):75-82.

33. Bertuzzi R, Franchini E, Kiss M. Análise da força e da resistência de preensão manual e as suas relações com variáveis antropométricas em escaladores esportivos. R Bras Ci e Mov. 2005;13(1):87-93.

34. Oliveira E, Da Silva V, Turquetto A. Relação do teste de caminhada pós-operatório e função pulmonar com o tempo de internação da Cirurgia Cardíaca. Rev Bras Cir Cardiovasc. 2009;24(4):478-84.

35. Watsford ML, Murphy AJ, Pine MJ. The effects of ageing on respiratory musclefunction and performance in older adults. J Sci Med Sport. 2007;10(1):36-44.

36. Renault J, Costa R, Rossetti M. Fisioterapia respiratória na Disfunção Pulmonar pós-Cirurgia Cardíaca. Rev Bras Cir Cardiovasc. 2008;23(4):562-9.

37. Silveira A, Sípoli L, Augusto V, Xavier M, Évora P. Comparação do uso da pressão positiva com a fisioterapia convencional e incentivadores respiratórios após cirurgia cardíaca. Medicina (Ribeirão Preto). 2011;44(4):338-46.

38. Blair E, Hickam JB. The effect of change in body position on lung volume and intrapulmonary gas mixing in normal subjects. J Clin Invest. 1955;34(3):383-9.

39. Druz WS, Sharp JT. Activity of respiratory muscles in upright and recumbent humans. J Appl Physiol Respir Environ Exerc Physiol. $1981 ; 51(6): 1552-61$.

40. Sibinelli M, Maioral D, Falcao A, Kosour C, Dragosavac D, Lima N. Efeito imediato do ortostatismo em pacientes internados na unidade de terapia intensiva de adultos. RBTI. 2012;24(1):64-70.

41. DATASUS. Sistema de Informações do SUS 2012 [Internet]. [Citado 16 de abril 2013]. Disponível em: http://www2.datasus.gov.br/ DATASUS/index.php?area $=02$.

42. Júnior S. A importância da Mobilização Precoce em pacientes internados na Unidade de Terapia Intensiva: Revisão de Literatura Perspectivas Online, Biológicas \& Saúde. 2013;10(3):15-23. 